\title{
Complementary value of functional capacity evaluation for physicians in assessing the physical work ability of workers with musculoskeletal disorders
}

\author{
Haije Wind • Vincent Gouttebarge • \\ P. Paul F. M. Kuijer · Judith K. Sluiter • \\ Monique H. W. Frings-Dresen
}

Received: 7 May 2007 / Accepted: 22 September 2008 / Published online: 9 October 2008

(C) The Author(s) 2008. This article is published with open access at Springerlink.com

\begin{abstract}
Objective To study the complementary value of information from functional capacity evaluation (FCE) for insurance physicians (IPs) who assess the physical work ability of claimants with long-term musculoskeletal disorders (MSD).

Method A post-test only design was used in the context of disability claims. Twenty-eight IPs participated in the study. Claimants with MSD formed the patient population. For each IP, the first claimant who agreed to participate was included in the study, and underwent FCE in addition to the regular disability claim assessment. Firstly, the IP performed the statutory disability claim assessment. Secondly, the FCE assessment took place. Finally, a self-formulated questionnaire was presented to the IPs after they viewed the FCE report. IPs were asked whether they perceived FCE information to be of complementary value to their judgment of the claimant's physical work ability investigated. We considered FCE information to be of complementary value if more than $66 \%$ of the IPs indicated as such. IPs were also asked whether FCE information led them to change their initial judgment about the claimant's physical work ability, and whether they felt this information made them more confident about their ultimate judgement. Finally, they were asked whether they planned to include FCE information in future disability claims and for what type of claimants. Differences between IPs who did or did not experience complementary value were explored.
\end{abstract}

H. Wind $(\bowtie) \cdot$ V. Gouttebarge · P. P. F. M. Kuijer .

J. K. Sluiter · M. H. W. Frings-Dresen

Coronel Institute of Occupational Health,

Academic Medical Center, University of Amsterdam,

P.O. Box 22700, 1100 DE Amsterdam, The Netherlands

e-mail: h.wind@amc.uva.nl
Results Of the 28, 19 (nearly 68\%) IPs considered FCE information to be of complementary value for their assessment of claimants with MSD. Half of the IPs stated that FCE information reinforced their judgment. All but four IPs changed their assessment after reading the FCE report. Sixteen IPs intended to involve FCE information in future disability claim assessments. There were no observed differences between the IPs who did or did not consider the FCE information to be of complementary value.

Conclusion FCE information was found to have complementary value at present and in the future according to most IPs in the assessment of the physical work ability of claimants with MSD. Half of the IPs felt that this information reinforces their judgment in this context.

Keywords Functional capacity evaluation - Work ability Insurance physician $\cdot$ Complementary value .

Musculoskeletal disorders

\section{Introduction}

Having work and being able to work are considered to be important requirements for being a full member of society. Work is an essential part of life for most of us. Inability to work, either because of unemployment, sickness or disability, has a negative impact on our quality of life (Van de Mheen et al. 1999). Interventions aimed at assisting people in getting back to work should thus be encouraged. The assessment of the ability to work can play an important role in this context by permitting differentiation between those who can work and those who cannot. The former can be helped to return to work, while the latter are entitled to a temporary or permanent disability pension. The assessment 
of work ability can thus have a major impact both on the individual and on society as a whole.

In the Netherlands, insurance physicians (IPs) receive a 4-year training in the assessment of work ability in persons who claim a disability pension after 2 years of sick leave. However, proper instruments for such assessment are lacking. The main source of information about the work ability of a claimant is the claimant him- or herself (De Bont et al. 2002). Since the claimant's opinion can differ considerably from that of the IP (Rainville et al. 2005), there is a need for additional information (e.g., from physical examination or from the claimant's own doctor or specialist) if the work ability is to be reliably assessed. Only a few instruments are available for assessing the physical work ability of claimants with a musculoskeletal disorder (MSD), and even these are only applicable to special groups of claimants (Wind et al. 2005). MSD is an important category of disorders in the context of disability claim assessments. In the Netherlands, about $30 \%$ of all disorders that led to disability claim assessments in 2004 involved the musculoskeletal system (Statistics Netherlands 2004). Musculoskeletal pain and its consequences are very common in the Dutch population of 25 years and older (Picavet and Schouten 2003). MSD is also an important cause of absenteeism and disability in the USA and other European countries, leading to a high national illness burden (Le Pen et al. 2005; Lubeck 2003).

Assessment of the physical work ability is a common practice in disability claim procedures. It is, however, a complex task, and IPs cannot rely on many instruments to support them in that task. Several studies indicate the weak relation between pathoanatomic findings and functional capacity (Tait et al. 2006; Vasudevan 1996).

One instrument that might help IPs to assess the physical work ability of claimants with MSD is functional capacity evaluation (FCE). This approach makes use of highly structured, scientifically developed, individualized work simulators, designed to provide a profile of an individual's workrelated physical and functional capabilities (Lyth 2001). According to Harten (1998), FCE offers a comprehensive, objective test that measures the individual's current functional status and ability to meet the physical demands of a current or prospective job. In particular, FCE provides information on physical work ability, being especially important in the assessment of disability in claimants with MSD and pain syndromes (Vasudevan 1996). The information of an FCE assessment can be used for several purposes, among which making disability determinations (King et al. 1998). Innes and Straker (1999a, b) reported the level of reliability and validity of several FCE methods and concluded that both for reliability as for validity adequate levels were lacking. However, in an update Innes (2006) concluded that since 1997 there had been a dramatic increase in the research in this field, with several FCEs showing moderate to excellent levels of reliability. FCE information offers a view on the ability to perform physical activities, which is an important part of the full work ability, especially in patients with MSDs. In a previous study, we found that IPs who assess claimants with long-term disability have mixed opinions on the utility of FCE (Wind et al. 2006). In fact, it appeared that only few physicians were familiar with FCE. Therefore, the topic of this study is whether FCE information can be of assistance to IPs in the assessment of the physical work ability of claimants, irrespective of their previous familiarity with the instrument. This is a first step in the process of possibly introducing FCE in the process of assessing disability claims of claimants with MSDs. More specifically, the questions to which an answer was sought are:

- Is information derived from FCE of complementary value for an IP in the assessment of the physical work ability of claimants with MSD?

- Are there differences between IPs who do or do not consider FCE information of complementary value in terms of personal characteristics of the IPs, themselves, or their claimants?

- Does FCE information lead IPs to change their assessment of a claimant's physical work ability, and/or does it reinforce their judgment, both in all IPs and in the subgroups of IPs who do and do not consider FCE information of value?

- After having been introduced to FCE, are IPs likely to make use of FCE information in the assessment of claimants in the future? If so, for what groups of claimants, and what differences exist between the groups of IPs who do versus do not consider the FCE information to be of complementary value for future use?

\section{Methods}

The present investigation was designed as a post-test only study.

\section{Participants}

\section{Insurance physicians}

A total of 100 IPs who assess claimants for long-term disability benefits were randomly selected from a pool of 566 IPs who work for the Institute for Employee Benefit Schemes (UWV) in the Netherlands. This semi-governmental organization employs all IPs who perform statutory assessments of claimants for long-term disability benefit in the Netherlands. To test the hypothesis that $66 \%$ of the IPs conclude that FCE information has a complementary value 
for the assessment of physical work ability, under the assumption of the $\mathrm{H}_{0}$ hypothesis of $40 \%$ (Wind et al. 2006), 28 IPs had to be included $(\alpha=0.05, \beta=0.8)$. All participating IPs signed an informed consent form.

\section{Claimants}

Each IP gave information about the study to a number of MSD claimants who were due to be assessed in the context of long-term disability benefit claims. The information packet included an application form that the claimant could fill out and send directly to the researchers. The claimants could also indicate that they did not wish to participate and explain why (though they were not obliged to give any reason). The first claimant seen by a given IP who agreed to take part in the study underwent an FCE assessment after signing an informed consent form. The claimant received a copy of the FCE report. The Medical Ethical Committee of the Academic Medical Center, Amsterdam, approved the study. The study period was from November 2005 to February 2007.

\section{Procedure}

Each IP was asked to assess the physical work ability in accordance with the statutory rules for the claimant who had volunteered to participate in the study. After receiving the report of the FCE assessment from the FCE provider, this report was presented to the IP in combination with his own report in the patient's file. After reading the FCE report, the IP was requested to fill in a questionnaire in which he gave his opinion of the complementary value of the FCE information and stated whether the information led him to change his initial assessment. The statutory assessment of the claimant for the purposes of the disability benefit claim was based on the IP's initial judgement, i.e., the FCE information had no influence on this statutory assessment (Fig. 1).

\section{FCE test}

The FCE instrument used in this study was the Ergo-Kit. This FCE is comprised of a battery of standardized tests that reflect work-related activities. The standard protocol, containing 55 tests, was performed by certified raters and took approximately three hours to complete. As a part of the test, claimants also filled in the Revised Oswestry Pain questionnaire (Hudson-Cook et al. 1989). All raters have followed a trainings program and are certified, and have to attend a refresher course twice a year. The Ergo-Kit lifting tests were found to be reliable in subjects both with and without musculoskeletal complaints with respect to the lifting tests (Gouttebarge et al. 2005, 2006). There is no information known to us from international literature about the reliability of the other tests of the Ergo-Kit FCE, neither is there information available about the predictive validity of this FCE. Claimants with a medical contra-indication for FCE, e.g., recent myocardial infarct, heart failure or recent surgery, were excluded from the test.

\section{Outcomes}

The questionnaire presented to all IPs contained three questions:

1. The IP was asked whether the FCE assessment had complementary value for the assessment of the physical work ability of the patient. The response choices were dichotomous: yes or no. With regard to the subquestion, characteristics of IPs and claimants that were believed to influence the answer of IPs about the complementary value of FCE information were classified. The characteristics selected for the IP group were work experience and familiarity with FCE. Work experience was found to be a factor that influences the way IPs come to their judgment about work ability (Razenberg 1992; Kerstholt et al. 2002). Familiarity with FCE was judged to be another reason why IPs might think differently about the complementary value. It was deemed possible that earlier contact with FCE information led to a negative opinion, as shown in the study about the utility of FCE information (Wind et al. 2006). The characteristics registered in the claimant group were the location of the disorder, their working situation, and functional disability. Location of disorders could be a factor for differences in judgment of the complementary value of FCE information. It is possible that FCE information could be judged as more valuable in assessments of claimants with general disorders than specifically localized disorders. Work status is another characteristic of the claimants that could lead to a difference between the group of IPs that considers FCE
Fig. 1 A flow diagram of the study design

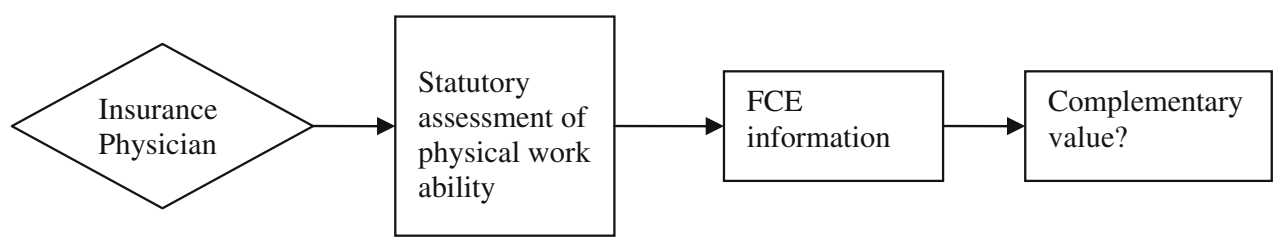


information to be of complementary value versus those that do not. The information that a claimant is currently working might make the information from an FCE assessment appear less valuable, and thus influence the IP's perception of the complementary value of FCE information. Functional disability was also assessed with the revised Oswestry questionnaire. The revised Oswestry questionnaire is derived from the Oswestry questionnaire (Fairbank et al. 1980) and is a 10-item instrument designed to measure the effects of pain on functional disability. Results of the revised Oswestry questionnaire were noted in numbers of claimants according to the five classes outlined by the revised Oswestry questionnaire: 0-20, 20-40, 40-60, 60-80, $80-100 \%$. A higher class indicates a higher level of functional disability.

2. (a) For each of 12 activities selected on the basis of a previous study (Wind et al. 2005) as representative of the physical work ability of claimants with MSD (walking, sitting, standing, lifting/carrying, dynamic movement of the trunk, static bending of the trunk, reaching, movement above shoulder height, kneeling/ crouching and three activities related to hand and finger movements), the IP was asked whether the FCE information caused him to revise his initial assessment of the claimant's ability upwards or downwards, or if it did not change the original assessment. (b) The IP was asked whether the FCE information had reinforced his initial assessment of the claimant's physical work ability. The response categories were, again, dichotomous: yes or no.

3. Finally, the IP was asked whether he would consider using FCE in the future to support assessment of the physical work ability of disability benefit claimants; and if so, why, and for what groups of claimants in particular. If he did not favor the use of the FCE, the IP could also state their reasons for this view.

Data analysis

Descriptions of IPs and claimants were calculated. Age and years of experience of IPs were expressed as mean and standard deviation (SD). The other characteristics of IPs, such as gender and familiarity with FCE, were noted in numbers and percentages. The age of the claimants was expressed as mean and SD. The distribution of the location of the MSD (upper extremity, lower extremity, back and neck, or more than one location) was noted using numbers and percentages.

The answer to the first question in the IP questionnaire (whether FCE information was regarded as having complementary value for the assessment of physical work ability) was scored as affirmative when at least $66 \%$ of the IPs answered yes to this question. Differences between the groups of IPs that did and did not consider FCE information to be of complementary value, were studied using independent $t$ tests for the relationship between work experience of IP and the outcome on the question about the complementary value of FCE information. Chi square tests were used to assess differences between the two groups-IPs who do and do not consider the FCE information to be of complementary value - on familiarity with FCE (IPs), location of disorder of the claimant, and claimant's work status. Kendall's tau-c was used to test the association between the two groups of IPs regarding the scores of the revised Oswestry outcome of the claimants.

For the answers to the question about the change in IP judgment based on FCE information, the numbers and percentages of IPs in the three categories (IP's assessment remained unchanged, increased, or decreased with respect to the claimant's abilities) were noted for each of the 12 activities. In addition, these data and their relation to whether the IPs did or did not consider the FCE information to be of complementary value were tested using Chi square tests. The outcome of whether FCE information had reinforced the judgment of physical work ability was scored affirmatively when $66 \%$ of the IPs answered 'yes.'

Answers to the third question were noted as the number and percentage of IPs answering 'yes' or 'no' with regard to their intention to use FCE in future assessments, along with the reasons given for this intention and the groups of claimants for which FCE information was considered to be particularly useful. Furthermore, differences between the group of IPs who did or did not consider the FCE information to be of complementary value were tested with reference to the intention of future use of FCE information using Chi square tests.

Finally, the relationship between the answers concerning complementary value and reinforcement of judgment and intention of future use were studied using independent $t$ tests. The significance level of all statistical tests was set at $P<.05$.

\section{Results}

Fifty-four IPs were prepared to take part in the study and signed an informed consent form, resulting in a response rate of $54 \%$. For 26 of these IPs, no claimant application forms were received within the study period and they were not included in the study. This left 28 IPs, each with one claimant with MSD whose physical work ability was assessed. Table 1 shows descriptive information of the study population. The mean age and SD of the IPs was 48 (7) years, and $64 \%$ of the IPs were male. Their mean experience (SD) in the assessment of disability benefit claimants 
Table 1 Gender (number, percentage), age in years (mean, SD), years of experience (mean, SD) and familiarity with FCE (number, percentage) of the insurance physicians $(N=28)$. Gender (number, percentage), age in years (mean, SD), and region of disorder (number, percentage) of the FCE claimants $(N=28)$

\begin{tabular}{lll}
\hline & $\begin{array}{l}\text { Insurance } \\
\text { physicians }\end{array}$ & Claimants \\
& $N=28$ & $N=28$ \\
\hline Men (number, percentage) & $18(64)$ & $11(39)$ \\
Women (number, percentage) & $10(36)$ & $17(61)$ \\
Age in years (mean, SD) & $48(7)$ & $46(5)$ \\
Experience in years (mean, SD) & $15(7)$ & \\
Familiarity with FCE (number, percentage) & $15(54)$ & \\
Region of disorder & & \\
Upper extremity (number, percentage) & & $3(11)$ \\
Lower extremity (number, percentage) & & $2(7)$ \\
Neck and back (number, percentage) & & $15(54)$ \\
Combination (number, percentage) & & $8(29)$ \\
\hline
\end{tabular}

was 15 years (7). Of the 28 IPs, 15 were familiar with FCE. Between the two groups of IPs, those whose claimants did or did not enter the study, no significant differences existed for age, gender, or years of work experience. The claimants of IPs who were familiar prior the study with FCE participated more often than claimants from IPs who were not familiar with FCE prior to the study $(P=.02)$.

Twenty of the claimants included were seen in the context of a disability re-assessment procedure, i.e., they were currently receiving a full or partial disability pension and were re-assessed pursuant to statutory requirements. The other eight claimants came for initial assessment of a disability claim after 24 months of sick leave. These 28 claimants were subjected to a standard Ergo-Kit test protocol by 13 certified raters at 13 locations throughout the Netherlands. Their mean years of experience were 4.5 years (median 5 years, SD 1.3 years).

The mean age (SD) of the claimants was 46 years (5) and $41 \%$ of the claimants were male. Of the 28 claimants, 15 had MSD of the neck and back, and eight had a disorder extending to more than one region. Upper and lower extremity disorders were reported in two and three claimants, respectively. For one claimant was reported that he had inconsistencies of test results and self limitation of performance.

\section{Complementary value}

Of the 28 IPs, 19 (68\%) indicated that FCE had complementary value for assessment of the physical work ability of the claimant under review. This percentage is greater than the stated threshold of $66 \%$. Only eight IPs gave voluntary a comment in addition to the response about complementary value. The tendency in the spontaneously given comments was that the complementary value of the FCE information was limited. Referring to the sub-question, neither work experience nor familiarity with FCE was significantly different between the group of IPs that did and did not consider FCE information to be of complementary value.

Change and reinforcement of judgment

The IPs indicated that they changed their judgment about the work ability of the claimants to perform the 12 activities because of the FCE information 127 (38\%) times. In 209 (62\%) times, the IPs indicated no change in their judgment. The number of changed judgments about the ability to perform the 12 activities was $108(47 \%)$ in the group of IPs that considered FCE information to be of complementary value $(n=19)$ and $19(18 \%)$ in the group of IPs that did not consider FCE information to be of complementary value $(n=9)$. Therefore, IPs that considered FCE information to be of complementary value changed their judgment more often than IPs that did not consider FCE information to be of complementary value $(P=.004)$. The numbers and percentages of IPs who changed their judgment after studying FCE information, and the direction in which the judgment was changed for the 12 activities in question, are presented in Table 2. Four IPs did not change their assessment for any activity. Neither on characteristics of IPs or patients, nor on reason for referral and FCE rater, differences were found between the group of IPs who did alter their judgment on one or more activities and the four IPs who did not alter their judgment on any of the activities. All IPs who did not alter their judgment on any of the activities considered the FCE information not to be of complementary value and had no intention of using this information in future disability claim assessments. On these two outcomes, these IPs differed significantly from the total group of IPs (Kendall's tau-b $P<.05)$. On average, IPs changed their assessment on four activities (mean 4.0, SD 2.6), with a range from 0 to 10 activities. Most IPs changed their judgment for lifting/ carrying, and moving above shoulder height. Out of 26 IPs, $15(58 \%)$ who indicated that they changed their judgment of the claimant's ability to lift and carry: seven IPs raising their estimate and eight IPs lowering it. Similarly, 10 out of 24 IPs indicated that they changed their assessment of the ability to work above shoulder height: eight IPs lowered and two IPs raised their estimate.

A majority of the $71 \%$ of the IPs (15 out of the 21 ) indicated that FCE information reinforced their judgments of physical work ability. This is more than the stated threshold of $66 \%$. Thus, we conclude that FCE information did serve to reinforce IPs' judgment in this study. Of the 15 IPs, 12 
Table 2 Total number of insurance physicians that assessed the activity, numbers and percentages of insurance physicians who changed their assessment of a claimant's ability to perform 12 different activities after studying FCE information, and the direction of this change

\begin{tabular}{lllll}
\hline & IPs & Change & More ability & Less ability \\
& $N$ & $N(\%)$ & $N(\%)$ & $N(\%)$ \\
\hline Walking & 26 & $9(35)$ & $6(67)$ & $3(33)$ \\
Sitting & 28 & $9(32)$ & $5(56)$ & $4(44)$ \\
Standing & 27 & $9(33)$ & $5(56)$ & $4(44)$ \\
Lifting/carrying & 26 & $15(58)$ & $7(47)$ & $8(53)$ \\
Dynamic trunk movement & 25 & $5(20)$ & $3(60)$ & $2(40)$ \\
Static bending trunk & 26 & $5(19)$ & $1(20)$ & $4(80)$ \\
Reaching & 26 & $7(27)$ & $1(14)$ & $6(86)$ \\
Moving above shoulder height & 24 & $10(42)$ & $2(20)$ & $8(80)$ \\
Kneeling/crouching & 25 & $9(36)$ & $1(11)$ & $8(89)$ \\
Repetitive movements hands & 24 & $6(25)$ & $3(50)$ & $3(50)$ \\
Specific movements hands & 25 & $5(20)$ & $3(60)$ & $2(40)$ \\
Pinch/grip strength & 25 & $6(24)$ & $3(50)$ & $3(50)$ \\
\hline
\end{tabular}

(80\%) from the IPs that considered FCE to be of complementary value and five of the eight IPs (63\%) that considered FCE information not to be of complementary value, indicated that the FCE information had reinforced their judgment. The difference between the two groups was not significant.

Future use

Out of the 28 IPs, 18 (64\%) indicated that they intended to use information from FCE assessments in future disability claim procedures. Out of 28 IPs, 20 (71\%) were positive about the complementary value of FCE information and 17 out of the 20 IPs $(85 \%)$ indicated that they intended to make use of FCE information in the future. Eight IPs were not positive about the complementary value of FCE information in their claim assessment. One of these eight IPs indicated that he intended to make use of FCE information in the future.

Arguments given in favor of FCE information were: the information is objective, it gives a better insight in the claimant's work ability, and it leads to better acceptance of the IP's decision by the claimant. Nine IPs reported these arguments. Arguments given against future use of FCE information, including the number of IPs who mentioned these arguments, were: the complexity of the FCE report $(n=2)$, the duration and cost of an FCE assessment $(n=4)$, the fact that FCE information does not make a distinction between restrictions in work ability based either on disorders or on personal traits $(n=2)$, and that inconsistencies in performance and sub maximal effort and observation of cooperation was thought to be possible $(n=2)$. The groups of claimants for which FCE information was thought to be useful were claimants with MSDs, claimants with medically unexplained disorders, claimants with complex disor- ders, which make it difficult to assess the work ability, like fibromyalgia, chronic fatigue syndrome, whiplash, and repetitive strain injury, and claimants with a large discrepancy between objective findings and subjective feelings of disability on one side and claimants with MSDs on the other side. These groups were named by resp. three and six IPs, respectively. Two IPs gave arguments in favor of FCE assessment not specifically related to claimant characteristics, like when the question about fitness for one's own job is at stake.

Complementary value and future use

Finally, IPs who indicated that FCE information has complementary value also have more often the intention of using FCE information in future disability claim assessments $(P=.01)$, confirming the hypothesis that a positive judgment about the complementary value of FCE was related to an intention of future use of this information in disability claim procedures. No relation was found between the answer about the complementary value and the reinforcement of judgment. This implicates that FCE information can reinforce the judgment about the physical work ability without being judged as of complementary value according to IPs.

\section{Discussion}

The aim of this study was to establish whether FCE information had complementary value for IPs in their judgment of physical work ability. About two-thirds of the IPs affirmed the complementary value of FCE in this context, and stated that it helped to provide a firmer basis for their decisions. Sixty-four percent of the IPs indicated that they 
intend to include FCE information in future disability claim assessments.

In contrast to earlier studies about FCE information in work situations (Gross et al. 2004; Gross and Battié 2004, 2006), this study took disability claim assessments into context. The strength of the study is that FCE information was introduced into the normal routine of disability claim assessments. This means that the IPs' judgment about the complementary value of FCE information was placed in the context of work ability assessment practice; it should be noted, however, that the FCE information did not influence the official judgment in the disability process. When an instrument is stated to have complementary value for IPs in the assessment of physical work ability, it should reinforce their judgment and/or alter their judgment of the physical work ability. A majority of IPs did, indeed, indicate that the FCE information had reinforced their initial judgment. Also, a majority of IPs altered their initial assessment as only four IPs stuck by their original appraisal of all activities considered. Three comments may be made in this regard:

1. Reinforcement of one's judgment does not necessarily exclude all changes in the assessment of individual aspects - an IP may well change his opinion about the claimant's ability to perform one or two activities while still feeling more confident in his initial appraisal of the overall physical work ability.

2. IPs did not change their opinion in any specific direction in this study. Roughly equal numbers revised their estimates upwards versus downwards. This is in contrast to the results of a previous study by Brouwer et al. (2005) that compared impairments in work ability as reported by the claimant, as assessed by the IP, and as estimated by FCE assessments. In that study, it was found that the self-reported level of impairment was highest, that derived from the judgment of IPs was at an intermediate level and that derived from FCE assessment was in general lowest, indicating that FCE would generally result in a downward revision of assessed impairment (Brouwer et al. 2005). The present study did not show such a shift towards higher work ability assessments (lower impairment assessments) after the IP had studied the FCE results.

3. No systematic connection was found between the location of the disorder (upper or lower extremity) and the reported changes in the assessment of performance. For instance, the ability to reach and perform activities above shoulder height, may be seen as a potential impairment in workers with upper extremity disorders, but was altered as well in claimants with disorders of the back or lower extremity.

To determine what factors might give cause to the opinion of some IPs that FCE information is of complementary value for the judgment of physical work ability in disability claim assessments, we examined differences between the groups of IPs that did and did not consider FCE information to be of complementary value. We analysed characteristics of both the IPs and of the included claimants. Work experience and familiarity with FCE were thought to be aspects that have influence on the outcome of complementary value of FCE. However, this did not appear to be the case. The other IP characteristics were not different, either. Although there was a difference in familiarity with FCE and participation of claimants in the study, there was no relationship between this finding and the outcome with regard to the question about complementary value, and therefore, the difference is not relevant to this question posed in the study. Another possible explanation for the difference between the two groups of IPs could result from a difference in their claimant population. Again, the different characteristics that were examined, location of disorder and work status, showed no significant differences between the two groups of IPs. The results of the revised Oswestry questionnaire had no relation with the judgement of the IPs about the complementary value of FCE. Therefore, it remains unclear why IPs have different opinions about the complementary value of FCE information.

The fact that FCE information is of complementary value increases the intention of future use. Thus, the hypothesis is not rejected that when IPs consider FCE information to be of complementary value, they will also intend to make use of this information in future disability claim assessments. One explanation for this might be that IPs do not have many instruments upon which to base their judgment when assessing work ability of claimants in the context of disability claims. FCE information is a potential instrument to assist them in this task. IPs in the group that considered the FCE information to be of complementary value, changed their judgment significantly more often as compared to their colleagues with the opposing opinion.

The following remarks may be made with regard to the external validity of the results:

1. In this study, IPs could not directly refer claimants for FCE assessment; moreover, claimants were completely free to decide whether they would participate and undergo the FCE assessment. This avoids the possibility of bias present in cases where claimants are referred to assessments like FCE by IPs. Since the IPs could not refer the claimants for FCE, their positive appraisal of the complementary value of such tests is unlikely to be falsified by their preconceived views.

2. Since a majority of the IPs indicated that they would consider using FCE information in future disability claim assessments, it may be expected that if they could refer claimants for FCE assessment in appropriate 
cases, their appreciation of the complementary value of FCE information might be even higher.

IPs believe that claimants for whom a discrepancy is found between the subjective complaints and expected objective findings would be a suitable target group for FCE in future disability claim assessments. In these cases, the claimant, who is usually the primary source of information (De Bont et al. 2002), will naturally tend to give a low estimate of their own physical work ability. The findings from physical examination, on the other hand, usually show little or no objective abnormality findings and cannot support the patients' view of their work ability. Whether this patient group is, indeed, a more suitable group for these forms of assessment of physical disability cannot be concluded from this study. This would, however, be an interesting topic for future research.

Some remarks are necessary about the choice of tests. In our study, we used the full FCE Ergo-Kit. Since the objective was to investigate the complementary value of FCE information for IPs in assessment of the work ability of claimants with MSD, there is no reason to limit the extent of the test battery. It is conceivable, however, that not all information generated by a full FCE may be required in all situations. It may not be relevant, for example, to assess the ability to kneel and crouch in claimants with impairments of the upper extremities. There have been requests for shorter FCEs, more specifically aimed at the work that the disabled worker is expected to do (Frings-Dresen and Sluiter 2003) or targeting the specific impairment in regional disorders (Gross et al. 2006; Soer et al. 2006). However, this study shows clearly that FCE information leads IPs to change their judgment even on activities not directly related to the underlying disorder and that IPs still regard this information as having complementary value. This is an argument for continuing the use of full FCEs. It is also noteworthy that the groups of claimants in whose assessment IPs indicated that FCE information would form a useful supplement largely presented problems of general physical functioning. Use of a full FCE would therefore seem to be called for in the assessment of such cases.

Finally, the practical implications of this study should be discussed. The positive evaluation of FCE information expressed by IPs in the study population argues for the introduction of FCE as a part of the disability claim assessment procedure, especially for those groups of claimants for which IPs think that FCE information yields maximum results. However, this study is based solely on the judgment of IPs towards the complementary value of FCE information. The prognostic value of FCE as a routine instrument in disability claim assessments has yet to be established.
Acknowledgments We would like to thank all functional capacity evaluation raters, insurance physicians and claimants who participated in this study.

Open Access This article is distributed under the terms of the Creative Commons Attribution Noncommercial License which permits any noncommercial use, distribution, and reproduction in any medium, provided the original author(s) and source are credited.

\section{References}

de Bont A, van den Brink JC, Berendsen L, Boonk M (2002) Limited control of information for work disability evaluation [De beperkte controle van de informatie voor de arbeidsongeschiktheidsbeoordeling: in Dutch]. Ned Tijdschr Geneeskd 146:27-30

Brouwer S, Dijkstra PU, Stewart RE, Goeken LN, Groothoff JW, Geertzen JH (2005) Comparing self-report, clinical examination and functional testing in the assessment of work-related limitations in patients with chronic low back pain. Disabil Rehabil 27:999-1005

Fairbank JCT, Couper J, Davies JB, O’Brien JP (1980) The Oswestry low back pain questionnaire. Physiotherapy $66: 271-273$

Frings-Dresen MHW, Sluiter JK (2003) Development of a Job-specific FCE protocol: the work demands of hospital nurses as an example. J Occup Rehabil 13:233-248

Gouttebarge V, Wind H, Kuijer PPFM, Sluiter JK, Frings-Dresen MHW (2005) Intra- and interrater reliability of the Ergo-Kit Functional Capacity Evaluation method in adults without musculoskeletal complaints. Arch Phys Med Rehabil 86:2354-2360

Gouttebarge V, Wind H, Kuijer PPFM, Sluiter JK, Frings-Dresen MHW (2006) Reliability and agreement of 5 Ergo-Kit Functional Capacity Evaluation in lifting tests in subjects with low back pain. Arch Phys Med 87:1365-1370

Gross DP, Battié MC (2004) The prognostic value of Functional Capacity Evaluation in patients with chronic low back pain: part 2; sustained recovery. Spine 29:920-924

Gross DP, Battié MC (2005) Functional Capacity Evaluation performance does not predict sustained return to work in claimants with chronic back pain. J Occup Rehab 15:285-294

Gross DP, Battié MC (2006) Does Functional Capacity Evaluation predict recovery in workers' compensation claimants with upper extremity disorders? Occup Environ Med 3:404-410

Gross DP, Battié MC, Cassidy JD (2004) The prognostic value of Functional Capacity Evaluation in patients with chronic low back pain: part 1; timely return to work. Spine 29:914-919

Gross DP, Battié MC, Asante A (2006) Development and validation of a short-form Functional Capacity Evaluation for use in claimants with low back disorders. J Occup Rehab 16:53-62

Harten JA (1998) Functional capacity evaluation. Occup Med 13:209_ 212

Hudson-Cook N, Tomes-Nicholson K, Breen A (1989) A revised Oswestry disability questionnaire. In: Roland M, Jenner JR (eds) Back pain: new approaches to rehabilitation and education. Manchester University Press, Manchester, pp 187-204

Innes E (2006) Reliability and validity of functional capacity evaluations: an update. Int J Disab Manag Res 1:135-148

Innes E, Straker L (1999a) Reliability of work-related assessments. Work 13:107-124

Innes E, Straker L (1999b) Validity of work-related assessments. Work $13: 125-152$

Kerstholt JH, de Boer WEL, Jansen EJM, Bollen D, Rasker PC, Cremer R (2002) Psychological aspects of disability claim assessment (Psychologische aspecten van de claimbeoordeling: in Dutch). TNO report, TM-02-C051, Hoofddorp, p 33 
King PM, Tuckwell N, Barrett TE (1998) A critical review of functional capacity evaluations. Phys Ther 78(8):852-866

Le Pen C, Reygrobellet C, Gérentes I (2005) Financial cost of osteoarthritis in France. The COART France study. Joint Bone Spine 72:567-570

Lubeck DP (2003) The costs of musculoskeletal disease: health needs assessment and health economics. Best Pract Res Clin Rheum 17:529-539

Lyth JR (2001) Disability management and functional capacity evaluation: a dynamic resource. Work 16:13-22

Statistics Netherlands (2004) Labour, income and social security (in Dutch). http://www.cbs.nl/theme

Picavet S, Schouten JSAG (2003) Musculoskeletal pain in the Netherlands: prevalences, consequences and risk groups, the $\mathrm{DMC}_{3}{ }^{-}$ study. Pain 102:167-178

Rainville J, Pransky G, Indahl A, Mayer EK (2005) The physician as disability advisor for patients with musculoskeletal complaints. Spine 30:2579-2584

Razenberg PPA (1992) Formation of judgment: scientific framework (Verzekeringsgeneeskundige oordeelsvorming: inzicht in de praktijk: in Dutch). PhD thesis, University of Amsterdam, Amsterdam
Soer R, Gerrits EHJ, Reneman MF (2006) Test-retest reliability of a WRULD functional capacity evaluation in healthy adults. Work 26:273-280

Tait RC, Chibnall JT, Andresen EM, Hadler NM (2006) Disability determination: validity with occupational low back pain. J Pain 7(12):951-957

Van de Mheen H, Stronks K, Schrijvers CTM, Mackenbach JP (1999) The influence of adult ill health on occupational class mobility and mobility out of and into employment in The Netherlands. Soc Sci Med 49:509-518

Vasudevan SV (1996) Role of functional capacity assessment in disability evaluation. J Back Musculoskel Rehab 6:237-248

Wind H, Gouttebarge V, Kuijer PPFM, Frings-Dresen MHW (2005) Assessment of functional capacity of the musculoskeletal system in the context of work, daily living, and sport: a systematic review. J Occup Rehab 15:253-272

Wind H, Gouttebarge V, Kuijer PPFM, Sluiter JK, Frings-Dresen MHW (2006) The utility of functional capacity evaluation: the opinion of physicians and other experts in the field of return to work and disability claims. Int Arch Occup Environ Health 79:528-534 